\title{
Crisis Management Exercises in Virtual Reality
}

\author{
Aurélie CONGÈS \\ IMT Mines Albi \\ Alexis EVAIN ${ }^{a}$ \\ IMT Mines Albi \\ Frédérick BENABEN \\ IMT Mines Albi \\ Olivier CHABIRON \\ Immersive Factory
Sébastien REBIÈRE IMT Mines Albi

\begin{abstract}
In crisis management, critical sites are required to carry out regular exercises to be prepared for a potential crisis. However, those exercises present a lot of drawbacks that reduce their efficiency: among other things, they are not frequent enough, not adjustable enough, too demanding and they are very expensive. The EGCERSIS project aims at using virtual reality to make fully configurable exercises to train in digital twins of actual critical sites. In this article, we present this project through a simple use case of a crisis in a subway station, involving three actors of crisis management.
\end{abstract}

Keywords: Virtual reality, training, coordination.

Index Terms: Human-centered computing-Human computer interaction (HCI)-Interaction paradigms - Virtual reality; Software and its engineering-Software organization and properties-Virtual worlds software-Virtual world training simulation

\section{INTRODUCTION}

Crisis management exercises in critical sites are indispensable and often obligatory. By critical sites, we refer to sites in which an incident or dysfunction can endanger people's life. To prepare for any type of crisis that can occur in the infrastructure, exercises are set up once or twice a year, or more or less frequently depending on the site and the legislation. However, exercises are complicated to set up and exploit, and they present limitations: for instance, they are expensive, time and resource consuming, they offer little realism and flexibility, as stated in [1].

To overpass those limitations, virtual simulations can be designed, like video games for instance. "Simulation-based training is commonly adopted in the field of aviation, military, and medicine" [2], and it helps trainees to gain a skill thanks to a safe and guided practice [3]. Those simulation-based exercises can be played thanks to keyboard and mouse or controllers, but we can also use them with virtual reality.

\footnotetext{
$\checkmark$ email: aurelie.conges@mines-albi.fr

email: alexis.evain@mines-albi.fr

^email: frederick.benaben@mines-albi.fr

* email: sebastien.rebiere-pouyade@mines-albi.fr

†email: ochabiron@immersivefactory.com
}

The EGCERSIS project proposes to create a training platform using virtual reality, and connected to a technological crisis management platform (TCMP).

This article is structured according to the following sections: first, an overview of the context, then, we present our virtual reality platform through a use case and an analysis of its real benefits. Finally, we list the next steps and perspectives.

\section{Context REgarding the domain of CRItical Sites PREPARATION}

The project EGCERSIS is based on the premise that current crisis management exercises in sensible sites are not as effective nor efficient as they could be and assumes that they would be improved by the use of virtual reality. This section aims at explaining why exercises are not effective or efficient enough. Then, we will explain how the project EGCERSIS could help.

\subsection{Drawbacks of current preparation exercises}

In most countries, critical sites are required to implement a set of measures like for instance, defining internal and external plans, and to run exercises to train the different actors (first and second aid and institutional responders such as firefighters).

According to [4], the exercises have a lot of drawbacks: "(i) traditional training simulated-based systems are expensive and offer little flexibility (e.g. it is not possible to simply burn up a building every time a drill is carried out); (ii) real training in the field of emergency management is very expensive and complicated regarding the harmonization of procedures between agencies". Besides, critical sites face several problems in the organization of crisis management exercises: mobilizing concerned actors is difficult, their implication and the impact it has on them is hardly predictable and may vary a lot. Mobilizing a site has economic impacts that have to be taken into account, and this means that it cannot be done very often. As a consequence, only a small number of crisis management scenarios can be run, which implies that it is impossible to cover every possible scenario and that the frequency of exercises is not high enough. Finally, the realism of the scenario is difficult to obtain, which affects the efficiency of an exercise.

\subsection{Virtual reality as a learning tool}

What we would like to emphasize in the definition of virtual reality is the notion of immersion: being there and being able to interact with the environment. As put by [5]: "A computer-generated system that generates an artificial world that tricks the user into feeling part of it would not be a complete definition of virtual reality because it lacks the vital component of interactivity. Virtual reality is a three-dimensional user interface in which the user can perform actions and experience their consequences." This point is crucial in our case because it will help the actors in the crisis management exercise to feel involved in it, and to really commit 
themselves to the resolution of the crisis. In their ten-year review of empirical research on the educational application of virtual reality, [6] show that "first order experience" and a sense of "presence" are key features from the technological characteristics of virtual reality that contribute to learning.

One of the identified drawbacks of the current crisis management exercises is the low frequency. As stated before, the disruption of the site as well as the mobilization of the actors, and the cost of the exercises, make them scarce. This implies that it is not possible to test every possible condition or scenario. Virtual exercises can represent a wide range of conditions while providing consistent and repeatable scenarios, each of them being practicable as many times as needed [7]. Besides, once the digital twin of the site and the scenario editor are made, it will not be any more expensive to use them multiple times rather than just once. Consequently, exercises will be easy to repeat. The presence of a scenario editor can also enable the exercises' designers to duplicate any condition. This means that it will not be necessary to wait for a rainy windy night in order to train for a crisis taking place in those conditions. This, joined to the repeatability of the exercises without any further cost, will help increase the coverage of the possible crisis for a critical site.

A virtual environment will also enable the actors of the exercise to try new ways of solving a problem, without being afraid of mistakes. "For training and education purposes, virtual reality allows trainees to practice cognitive, and sometimes physical, skills in simulation as risks to safety and material costs can be much lower in VR than in physical training" [8]. For instance, in the medical field, it will be possible to learn a certain surgery technique without risking to hurt the patient if it goes wrong. In crisis management, it could be possible to try new tools or new intervention plans to check their effectiveness and relevance. As put by Saunders et al. [9], in virtual reality exercises, "players are encouraged to learn by trying different strategies, succeeding and failing as they would in an entertainment game".

Gallerati [10] summarizes the advantages of virtual reality as a training tool in its paper "the use of virtual reality and simulators for emergency management". Training increases employees role awareness, commitment, and information retention while helping companies to stay compliant: "The use of simulators can help a lot in communicating emergency procedures to a wide range of people, by challenging them in a variety of foreseeable crisis situations without running the risk of exposing them to real risks before they are fully aware and trained, with the further benefit of achieving, through immersive situation, better retention of information received".

Another advantage that can be seen in virtual reality, besides all the previously seen, is the ability of virtual reality to go beyond reality. While the previous points are more about copying the reality, we can also use virtual environments to "extend the user's capability to feel data that would normally be beyond the range of their senses or experiences" [6]. The first idea coming from this point would be to use virtual reality as a new tool for data visualization and data analysis. Millais et al. [12] show in a study that "participants found data exploration to be more successful and satisfying in VR than in 2D", this being due to the "wider field of view, increased dimensionality and sense of presence" of virtual reality. The second idea coming from this point is to use virtual reality to enable an entirely new way of learning. As put by Lester in [9], "if you want to teach biology, why build a virtual classroom with desks and a blackboard in Second Life when you could build a whole interactive human cell?" This, added to the scalability of virtual reality, can give 'the users the ability to 'change' their physical size, so that they can navigate and interact in macro and micro worlds" [11].

Up to now, the main drawback of virtual reality is technologic, with the haptic rendering that is still weak. Even if head-mounted devices help the player being immersed in the virtual environment, it is still difficult to be able to make him feel resistance. This can be a problem for instance for medical training when you can't practice the feel of the resistance of the skin. In crisis management exercises, this can limit the realism of some scenarios, involving for instance casualties, or for felling the pressure in a fire hose. Nevertheless, virtual reality for learning stays at least as effective as traditional training, as shown by [13] in their paper where they compare several studies and conclude that "professional trainings can be fruitfully enhanced by VR".

\section{OUR PROPOSAL: THE EGCERSIS PROJECT}

The EGCERSIS project has been launched in April 2019 and should last three years. It aims at providing a framework dedicated to preparation and training for crisis management on critical sites. This is going to be achieved thanks to virtual reality and decision support, providing an immersive experience in a digital twin of the site (thanks to virtual reality goggles), linked to monitoring and supervision tools to generate reports to capitalize on the exercises.

The objectives of the project are going to be achieved mainly through the implementation of four components:

- $\quad$ Exercise editor (modeling and scenario definition)

- Virtual environment (multi-player scenario enactment)

- Decision support tools (accompanying decision-maker of the crisis cell)

- Dashboard tools (real-time and later analysis of players, exercise and plans).

Through these components, we propose to offer solutions to the drawbacks identified in real-life exercises.

Through the virtual environment, we plan on improving the quality of the exercises. This virtual environment is supposed to be a digital twin of the site in which the exercise has to take place. Thanks to virtual reality, we want the players to be able to evolve in the digital twin and to interact with the environment.

Thanks to the decision support tools, which are presented in $\{$ Citation $\}$, it will be easier to evaluate the plans and the response to the crisis. They will help gather data, using, for instance, several sensors, and analyze the data to integrate it into the response process. That gathered data will also be easy to analyze after the exercises, which will help capitalize on the exercises.

Dashboard tools will help capitalize and analyze the data and being able to establish lessons learned, on the crisis management plans as well as on the exercise.

The EGCERSIS research program will perform an iteration of several steps, which are presented in Figure 1. 


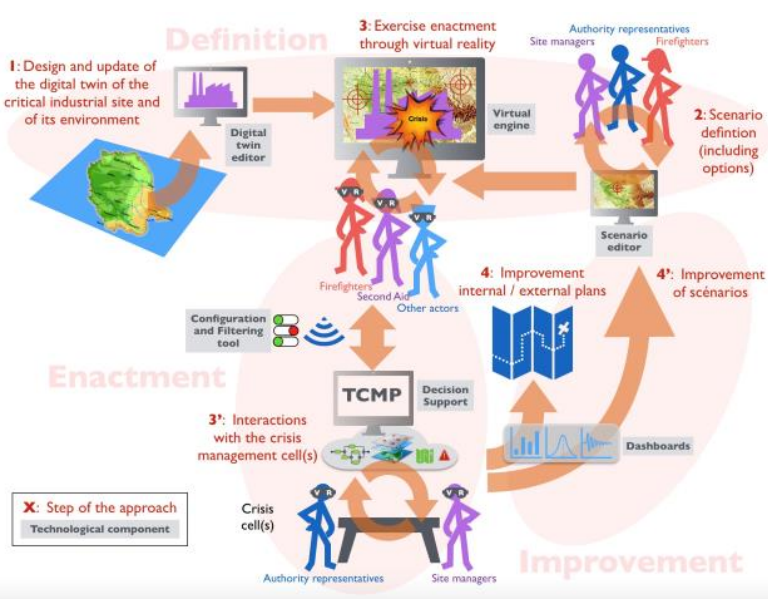

Figure 1: Big picture of the EGCERSIS project.

The first step will be to create a 3D digital twin of the site where the exercise will take place. This will be done thanks to plans of the buildings or maps for instance. The virtual environment, as a digital twin of the critical site, will not damage the realism of the exercises, since they will keep on taking place inside the concerned site. This will be possible thanks to formalized protocols for the establishment of digital twins of the sites, that will be set up within the project. It might even improve the realism since the crisis will be real inside the virtual environment. For instance, it is possible to implement a fire in the virtual site, or a flood or various types of crisis.

Then, a selected scenario will be implemented in the scenario editor. It will be used by site managers, responders or authority representatives to create a realistic scenario, that will be evolving throughout the exercise, depending on the responses of the actors.

Thirdly, actors of the crisis management in the chosen site will face the scenario and play the exercise. This will be done immersed in the virtual environment. Making sure that the actors can have access to realistic virtual tools, identical to those they use in a real crisis, is also one of our priorities since it would make no sense for them to train with tools that they would never use in real life. This is also designed to ensure the realism of the virtual exercises proposed by our platform. Meanwhile, crisis managers will deal with the crisis using our decision support tool.

Finally, all the data from the exercise will be recorded in realtime, which is made possible by its numerical nature. This means that everything can be monitored, or replayed, in order to analyze the exercise and get feedback. This should have two positive consequences, the first one being the improvement of the exercise, and the second one being the improvement of the crisis management in itself, including the intervention plans and the actors' skills.

\section{ILLUSTRATION WITH A SIMPLE USE CASE}

In this section, a use case that we set up in order to prove the advantages of using virtual reality in crisis management exercises is presented.

\subsection{Presentation of the use case}

Nota Bene: The following example is fictional and is not meant to be realistic. This simple use-case is dedicated to illustrating the previous section.

It is 8:30 am on the 28th of October 2019. A fire just declared in the Madeleine metro station. The station has to be evacuated and a security perimeter must be set up. An injured man is lying on the floor of the platform and has to be taken care of. Three actors are on the field to deal with the crisis: there are a fireman, a medical doctor, and a policeman.

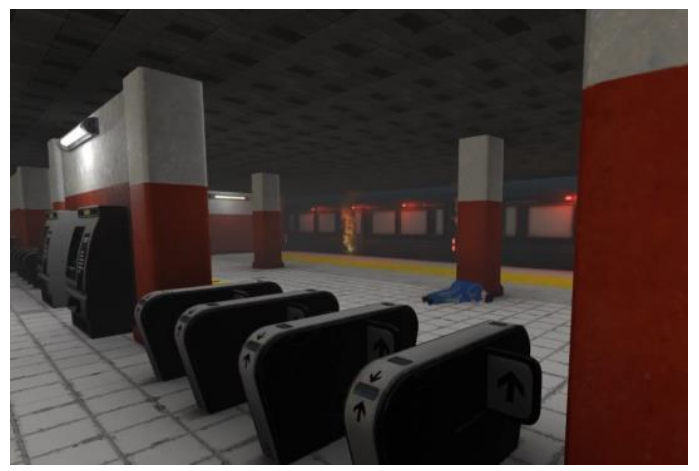

Figure 2: Madeleine metro station in the virtual environment.

Two versions of this use-case are played. In the first one, the players only have the information given above, and they have to fulfill their objectives. Each of the actors has access to a certain tool depending on what he has to do: the fireman has a fire hose, the medical doctor has a first aid kit with colored cards used to indicate the severity of the patient state, and the policeman has a ribbon to place on the doors to secure the perimeter.

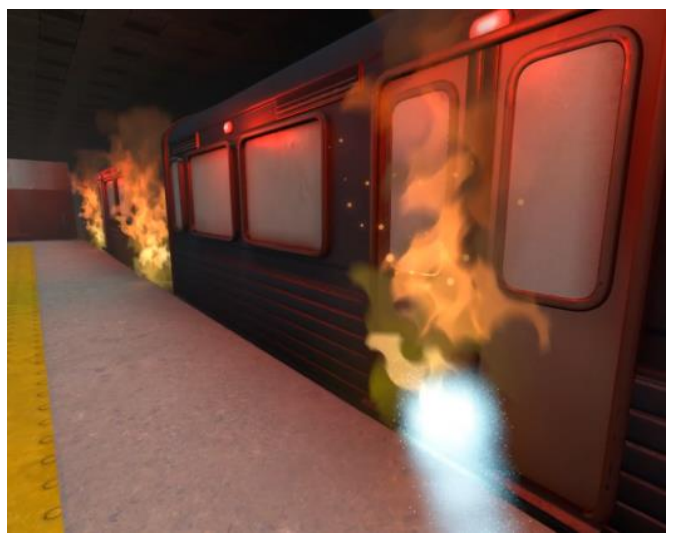

Figure 3: Fireman extinguishing a fire.

The second version of the use-case is played in connection with our TCMP. The platform is connected to virtual sensors and gathers and analyses the data coming from those sensors. That data is taken into account in the deduction of the process of crisis management proposed by the platform. A presence sensor detects the presence of a forgotten child on the other side of the platform, invisible from where the actors initially are. In the first version of the scenario, it is easy to miss him because he doesn't move nor make any noise, so if the actors don't take the initiative of searching the whole metro station, they won't find him. Thanks to the TCMP and its ability to analyze every data from any sensor, it is impossible to forget the child since a new task will be created in the process, asking the medical doctor, for instance, to go take care of him.

\subsection{Technical aspects}

This simple use-case is the first of the EGCERSIS project. The objective is to get familiar with the technologies and provide a first proof that realism and a sense of immersion are possible to achieve in virtual exercises. The actors of the exercise are evolving in a 
virtual environment built with Unity ${ }^{\circledR}$, in its version of 2019 . It has the advantage of being quick to master and allows you to have prototypes sufficiently advanced for research projects. The interaction with the environment is made with HTC Vive Pro ${ }^{\mathrm{ii}}$ virtual reality headset and controllers. Up to now, the movement is achieved thanks to teleporting only, but one of our next steps is to enable the movement to be more realistic thanks to the use of Kat VR ${ }^{\text {iii }}$ omnidirectional treadmills.

Several difficulties had to be overpassed in this first scenario: dealing with virtual reality obviously, including a multiplayer experience, and connecting our TCMP with the virtual reality environment.

In this first use case, we create a first version of a multiplayer mode, where the different actors are able to evolve in the same virtual environment and see each other. To do so, we use Photon Network ${ }^{\circledR i v}$ for its advanced state of game customization and connection between users. This system uses its own hosting network, which saves users from a whole panel of configuration to be deployed to be together in the simulation. For this use case, the three required players stand in the same room, as shown in Figure 4 , however, the players don't have to use the same connection, and can be at different locations places at a time.

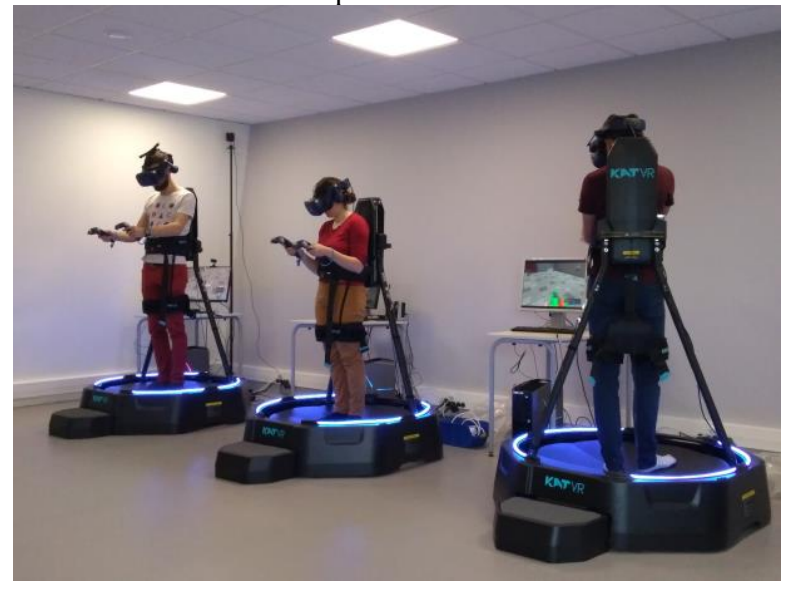

Figure 4: Players equipped with controllers and headsets.

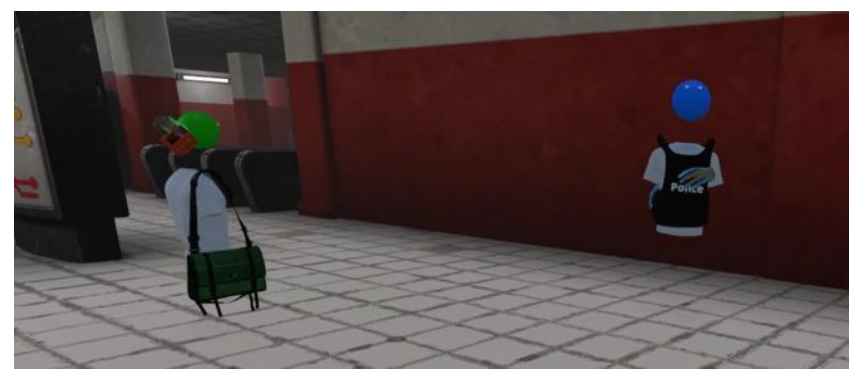

Figure 5: Avatars of the other players as seen by the fireman.

We also used Steam VR ${ }^{\mathbb{v}}$, which allows us to quickly deploy virtual reality for a player in a virtual environment. This technology has the advantage of being polyvalent and can be used with various equipment of virtual reality. For this simulation, we used wired and non-wired HTC Vive Pro ${ }^{\circledR}$. We choose this equipment for its digital rendering and its lightness. Figure 6 presents the links implemented to achieve multi-playing in the use-case. A first executable is implemented in Unity. Information on the positions, moves, and actions of the player are sent to Photon, which hosts the game and sends the data to the other players evolving in the virtual environment. At any time, the information of each player is communicated to all other players via Photon ${ }^{\circledR}$.

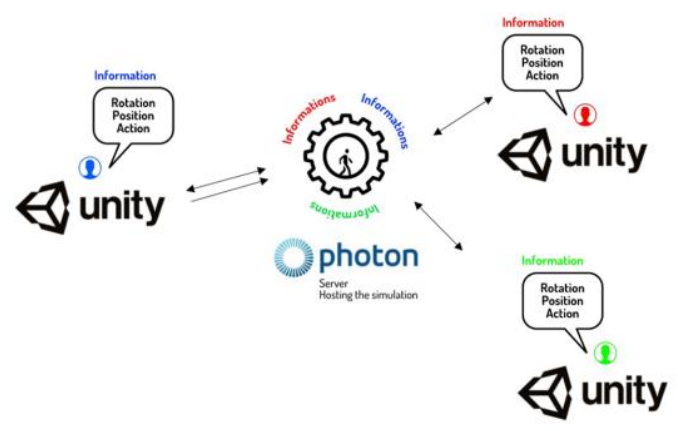

Figure 6: Architecture of the multiplayer aspect.

At the beginning of the second version of the use case, the players have to $\log$ in to our TCMP. This decision support tool orchestrates and organizes the different tasks that have to be fulfilled to solve the crisis in a BPMN process. Extinguishing the fire is attributed to the fireman, the policeman has to find the child and secure the perimeter, and the medical doctor has to deal with the wounded. The idea is not to tell the actors how to do their job, but to help them cooperate most effectively. It's all about coordination and not interference. The TCMP is able to follow the evolution of the crisis in real-time, and to detect differences between the actual situation and what the situation should be, had the process been followed. It can then adapt the process and send new tasks to the actors on site.

When it comes to planning an exercise, one of the important points is its modularity. In this first development, we established several configurable points, mostly from the player's point of view. The first choice that you have is the role of the actor that you want to play, along with the different functionalities that come with the role: do you want to train as a fireman, a policeman, a medical doctor? We can also decide to move the position of the objectives in the virtual exercise: where will be the fires to extinguish or the casualties for instance. It is also possible to randomize the appearance of the objectives so that it will appear in a random position every time you start the exercise.

\subsection{Analysis of the exercise}

This exercise and the two scenarios that have been used can be considered from different points of view. Basically, the analysis of the results can be described according to the following dimensions: (i) appropriation, (ii) demonstration, (iii) interaction:

- Appropriation: This dimension is certainly the most important of the expectation. Did the user improve their practical knowledge after performing the scenarios in the virtual reality environment? Basically, several "naïve" benefits can be identified: the first one is the "banalization of the exceptional", which means that, through repetitions, seeing a fire, hearing the sounds (sirens, fire, firehose, etc.), facing casualties, etc. is no longer an obstacle to performing appropriately. The second one concerns the efficiency and the fact that professional protocols, processes, and rules can be taught and learned through that kind of scenario (using close-to-real experience channels). The third one concerns the usage of virtual reality as a vector for continuous improvement. After a few 
experiments, virtual reality becomes a familiar environment (wearing the goggles, stepping on the walking platform, using the joysticks, etc.) in which it is easy to include new practices, new elements and new processes that will be more easily learned and assimilated by people due to the familiarity of the environment.

- Demonstration: This dimension concerns the fact that some tools, practices, or approaches can be studied and validated through that kind of exercise. For instance, the usage of sensors dedicated to monitoring vital signs of first responders is often mentioned as an avenue for the future equipment of first responders. However, the question of the concrete exploitation and visualization of that data is still pending. Simulating that kind of exercise, including virtual sensors for vital signs, could be a way to show if such an innovation could be fruitful and at which conditions (what kind of visualization not to overload the decision-makers). In the use-cased presented in this article, we are showing the relevance of using the TCMP in a crisis management exercise.

- Interaction: In this version of the use-case, the interaction feature only concerns two aspects: (i) coordination (the three actors being in the same scene, seeing each other, being able to coordinate their action) and (ii) use of tools (actors can use tools like firehose, enclosure tape, etc.). Other interaction aspects like (iii) use of the environment (players being able to open doors, move objects, etc.), or (iv) cooperation (actors being able to work simultaneously and conjunctly on specific tasks that would require several actors to be performed) have not been implemented. However, considering the next steps of the EGCERSIS project, these two features are clearly in the roadmap.

Finally, it is crucial to explicitly notice that the two phases scenario presented previously is clearly disconnected from reality. It is full of approximations and mistakes regarding the real practices of professional responders. For instance, it is almost certain that they would have the obligation of exploring entirely the area and would discover the additional victim without requiring the ability of the TCMP to interact with sensors. Another example concerns the types of responders. It is almost certain as well that there would not be only three of them, investigating at the same time, etc. However, on the one hand, this is mainly a proof of concept (showing that heterogeneous actors, using an external decision support tool could interact in a virtual environment to take care of individual and shared objectives), and on the other hand, the main purpose of the exercise was not to be close to reality but to address some technical and scientific challenges (especially on the previously mentioned dimensions) and to open doors for the next steps of the project.

\section{Next Steps}

We plan to release several use cases within the framework of the EGCERSIS project, each of them being more complex and more realist than the one before. To achieve that, we have several objectives that we want to fulfill, as exposed in this section.

First of all, we want to transpose our fictional scenario into a real use case, in a real site. We already have a digital twin of the building of our lab in our university, which we plan to use to train the security teams of the school (see Figure 7 and Figure 8). We also plan to do several other digital twins of partner companies within the project.

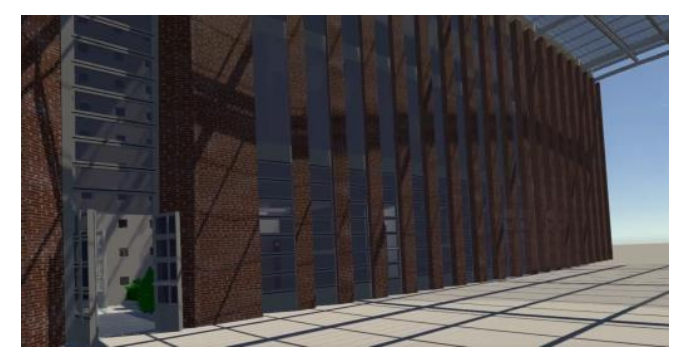

Figure 7: View of the digital twin of our university.

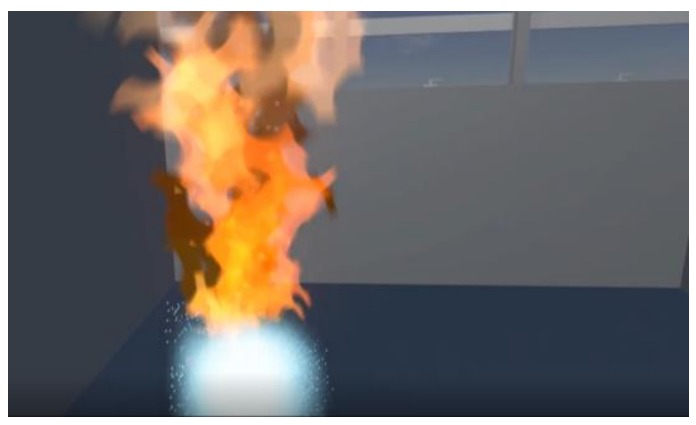

Figure 8: Fire in the digital twin of our university.

We would like to make it possible for the players to collaborate for the fulfillment of an objective, this for the exercise to be as realistic as possible. Currently, we only have three actors in the field, but it is not realistic. In a real crisis, a fire might take more than just one fireman to be put down; a casualty might have to be evacuated on a stretcher, which is impossible for a single man to do. So, we want to make interaction possible between the players, so that they won't only be able to see each other anymore, but also to realize actions together that cannot be done alone.

We plan on improving realism, for instance allowing the actors to walk instead of teleport themselves. To do so, we have to connect the Kat VR ${ }^{\circledR}$ treadmills to our virtual environment.

Another aspect of the project will be to enable the exercise designer to change the scenario while it is being played. For instance, the designer can decide to change the weather condition in the middle of the scenario, or to create a new start of the fire in another place, or to add new casualties, etc. Those new events of the crisis will then have to be taken into account by the TCMP which will deduce a new process for the actors.

The modularity is also to be achieved thanks to a scenario editor which will allow the exercise designer to build a large number of scenarios. Through a configurator, he will be able to choose the type of the crisis (fire, flooding, explosion, chemicals leak, etc.), the number and position of casualties, the time of the day and the duration of the exercise, the weather... We also want to enable the scenario designer to propose a multi-agent exercise, for instance using non-player characters. By multi-agent, we mean either malevolent actors such as terrorists for instance, or victims, running crowds, agents that are not really acting on the crisis but whose actions have to be taken into account or dealt with. This will help to achieve greater coverage of the different types of crisis that can happen in a given critical site.

In the end, the virtual environment that we want to provide will be full of several sensors of every kind: presence, location, heat, water level... However, depending on the crisis you are dealing with and the sensors actually available in the site, some of the virtual sensors might be useless or giving information that is not actually available. In order to not train to rely on data that won't be 
available in a real crisis, we need a way to stop that data from being taken into account in the training sessions. To do so, the EGCERSIS project plans to provide a filtering tool allowing the game designer to pick the sensors and the flows of data that are actually useful or available for a given crisis in a given site.

We also want to offer the practitioner the possibility to test new tools, by implementing them in the virtual environment. If the tool works the same in the real environment and the virtual environment, and if its impact on the virtual world is realistic enough, then we would be able to prove its usefulness. This could help determine if an investment is worth it or not.

Finally, the project strives to use virtual reality's ability to exceed the reality, for instance in the field of data visualization. We want to create a virtual environment dedicated to crisis management, for crisis managers and decision-makers, in which we want virtual reality to be a central tool in situation awareness and decision support.

\section{CONCLUSION}

The presented approach aims at using virtual reality to improve the efficiency and effectiveness of preparation exercises for critical sites' crisis management. The first profit of this approach is to have access to training in a realistic situation of an entirely configurable crisis. The second profit is the possibility to interface the exercise with emulated or existing tools (such as decision support tools) in order to determine their relevance. Practices and procedures will be easier to assimilate thanks to training in virtual reality than through theoretical learning.

In the next steps of the project, we want to expand our proposal along with three steps. The first one will be to improve the realism of the user experience thanks to the implementation of a scenario editor that will allow the scenario to be configurable, both before and during the exercise. We also want to set up a protocol to digitalize the critical sites.

The second step will concern playability. In the use-case presented in this article, the multiplayer aspect is reduced to each player being able to see the others. We want to improve this to make collaboration and cooperation possible. On the one hand, we want players to be able to do actions together. On the other hand, we want the players to be able to interact with their environment, with objects or tools, or non-player characters that can be either passive (such as wounded) or active (such as crowds or malevolent actors).

Beyond the training environment destined to crisis first responders, we want to provide a piloting environment for crisis managers and decision-makers, also using virtual reality instead of 2D screens. This will be our third step: using virtual reality for situation awareness and decision making. Our idea is to provide a virtual twin of the crisis, allowing the crisis managers to have both a global view of the situation and the ability to safely walk inside the site where the crisis takes place. The idea is to zoom in and out of the site in order to get multiple points of view of the situation. We also want to use virtual reality to go beyond reality and offer better tools for data visualization, situation awareness, and decision making.

\footnotetext{
${ }^{\mathrm{i}}$ https://unity.com/fr

ii https://www.vive.com/fr/product/vive-pro/
}

\section{References}

[1] L. Ardila, I. Pérez-Llopis, C. E. Palau, et M. Esteve, « LVC Training Environment for Strategic and Tactical Emergency Operations », in ISCRAM, 2013.

[2] F. Benaben et al., " A conceptual framework and a suite of tools to support crisis management », in HICSS 2017-50th Hawaii International Conference on System Sciences, 2017, p. 237.

[3] A. Conges, F. Benaben, O. Pierre, F. Savic, O. Chabiron, et M. Lauras, « On the usage of Virtual Reality for Crisis Management exercises in Critical Industrial Sites », in Proceedings of the Sixteenth International Conference on Information System for Crisis Response and Management, Valencia, Spain, 2019.

[4] L. C. Dukes et L. F. Hodges, « Development of a scenario builder tool for scaffolded virtual patients », in 2014 IEEE Virtual Reality (VR), 2014, p. 131-132.

[5] P. Gallerati, M. Maisano, C. Valiante, A. Conte, et D. Casciaro, «The Use of Virtual Reality and Simulators for Emergency Management Training Increases Employees' Role Awareness, Commitment and Information Retention, While Helping Companies to Stay Compliant. », présenté à Offshore Mediterranean Conference and Exhibition, 2019.

[6] S. Kang, J. Chanenson, P. Ghate, P. Cowal, M. Weaver, et D. M. Krum, «Advancing Ethical Decision Making in Virtual Reality », in 2019 IEEE Conference on Virtual Reality and 3D User Interfaces (VR), 2019, p. 1008-1009.

[7] P. K. Kwok, B. K. P. Chan, et H. Y. K. Lau, « A Virtual Collaborative Simulation-based Training System », in Proceedings of the 10th International Conference on Computer Modeling and Simulation, New York, NY, USA, 2018, p. 258-264.

[8] F. Lateef, « Simulation-based learning: Just like the real thing », J. Emerg. Trauma Shock, vol. 3, nº 4, p. 348-352, 2010.

[9] D. Livingstone, Éd., Proceedings of the First Second Life Education Workshop, part of the 2006 Second Life Community Convention, August 18th - 20th 2006, Fort Mason Centre, San Francisco, Ca. Paisley, UK: Univ, 2006.

[10] M. Louka et C. Balducelli, « Virtual Reality Tools for Emergency Operation Support and Training », p. 10.

[11] T. A. Mikropoulos et A. Natsis, «Educational virtual environments: A ten-year review of empirical research (1999-2009) », Comput. Educ., vol. 56, nº 3, p. 769-780, avr. 2011.

[12] P. Millais, S. L. Jones, et R. Kelly, « Exploring Data in Virtual Reality: Comparisons with 2D Data Visualizations ", in Extended Abstracts of the 2018 CHI Conference on Human Factors in Computing Systems, New York, NY, USA, 2018, p. LBW007:1-LBW007:6.

[13] J. Saunders, S. Davey, P. S. Bayerl, et P. Lohrmann, "Validating Virtual Reality as an Effective Training Medium in the Security Domain ", in 2019 IEEE Conference on Virtual Reality and 3D User Interfaces (VR), 2019, p. 1908-1911.

\footnotetext{
iii https://www.katvr.com/

iv $\underline{\text { https://www.photonengine.com/ }}$

v $\underline{\text { https://store.steampowered.com/steamvr?l=french }}$
} 